\title{
Evidence for two kinds of low-typical instances in a categorization task
}

\author{
ARNOLD L. GLASS and PETER J. MEANY \\ Rutgers University, New Brunswick, New Jersey 08903
}

Three experiments examined the relationship between instance typicality and reaction time (RT) in a semantic categorization task. In all three experiments, first the instance was presented, and then the category. High-typicality high-imagery instances (e.g., robin) and lowtypicality low-imagery instances (e.g., grackle) were categorized faster than low-typicality highimagery instances (e.g., penguin). Instructing subjects to generate images of the instances had no influence on the pattern of results. The difference in categorization RT for lowimagery low-typicality instances vs. high-imagery high-typicality instances suggests that these instances may be represented differently in memory.

A common finding in semantic categorization tasks is that the typicality of the instance (e.g., robin) to the category (e.g., bird) is correlated with the reaction time (RT) to categorize the instance as a member of the category. For true categorizations, that is, trials in which the instance is a member of the category, it is commonly found that more typical instances (e.g., robin) are categorized more quickly than less typical instances (e.g., penguin) (Rips, Shoben, \& Smith, 1973; Rosch, 1973).

The purpose of this study was to examine the relationship between instance typicality and RT for a true category judgment. The cause of this effect has been of considerable interest. McCloskey and Glucksberg (in press), Schaeffer and Wallace (1970), and Smith, Shoben, and Rips (1974) have all suggested that hightypicality instances are categorized faster because their semantic descriptions are more similar to that of the category than are the descriptions of low-typicality instances. According to this hypothesis, the more similar the instance and category descriptions are, the less time that is necessary to compare them before any affirmative judgment is made. This hypothesis will be called the comparison model of categorization, since typicality is assumed to influence the comparison stage of the categorization process.

In contrast, Collins and Quillian (1969) and Glass and Holyoak (1975) have suggested that high-typicality instances are categorized faster because information relevant to the decision can be accessed faster for them than for low-typicality instances. This hypothesis

This research was supported by National Institutes of Health Grant R03MH-28225 to Arnold L. Glass and by the Rutgers Research Council. We would like to thank Steve Sarafconn for preparing the items and testing the subjects for these experiments. Reprint requests should be sent to Arnold Glass, Rutgers College, Department of Psychology, Busch Campus, New Brunswick, New Jersey 08903 will be called the retrieval model of categorization because it assumes that typicality influences the stage of the categorization process when information about the instance is retrieved.

The motivation for this study was the speculation that both of these models are correct and that there are two kinds of low-typicality instances. For example, consider the category "bird." Some instances might receive low-typicality ratings because they access some property deviant from the category (e.g., penguins and ostriches do not fly). These instances would take longer than typical instances to categorize because it would take longer to compare their descriptions to that of the category. Other instances might receive low-typicality ratings simply because the rater does not know much about them other than the category of which they are members (e.g., warbler, grackle). These instances would take longer to categorize than typical instances because it would take longer to access relevant information about them.

Hence, this analysis suggests that there are not two kinds of instances (high vs. low typicality), but three. High-typicality instances (e.g., robin) are closely associated to a semantic description similar to the category's description. One kind of low-typicality instance (e.g., penguin) is closely associated to a description that is not similar to the category's description. Such an instance will be called an "atypical" instance. The other kind of low-typicality instance (e.g., grackle) is remotely associated to a description that identifies the category of which it is a member, but contains little or no additional information. Such an instance will be called an "unfamiliar" instance. Thus, this model assumes that some low-typicality instances are categorized more slowly than typical instances because it takes longer to retrieve their descriptions, while others are categorized more slowly because it takes longer to compare their descriptions 
to that of the category. This will be called a "mixed model" of semantic categorization. The following three experiments tested some of its predictions.

\section{EXPERIMENT 1}

In order to demonstrate that instances are categorized differently, it was first necessary to distinguish the three kinds of instances objectively. In order to do this, instances were selected on the basis of how they were rated along two dimensions: typicality and imagery. The use of typicality as one dimension was, of course, essential, since the question of interest was how instance typicality is related to categorization RT. The choice of imagery as the other dimension was based on pilot studies that revealed it was the best measure of how much a subject knew about an instance. Other measures tended to be contaminated by estimates of the instance's frequency in the local environment. For example, when asked to rate instances on the basis of familiarity, subjects tended to rate "warbler" higher than "penguin," al though, when asked to list a warbler's specific properties, most subjects revealed they knew nothing about it beyond the fact that it was a bird. In comparison, "penguin" received a significantly higher imagery rating than "warbler." Hence, first, a large number of instances for different categories were rated by two normative subject groups on imagery and typicality. Three kinds of instances were identified: high-typicality high-imagery instances (e.g., robin), low-typicality high-imagery instances (e.g., penguin), and low-typicality low-imagery instances (e.g., grackle). It was hypothesized that both high-imagery hightypicality instances and high-imagery low-typicality instances accessed their own descriptions, while the lowimagery low-typicality instances accessed only the category's description. That is, the only thing most people would know about the low-imagery lowtypicality instances would be the categories of which they are members. Hence, when asked a question about such an instance, the only decision strategy available to most subjects would be to access the description of the category of which the unfamiliar instance was a member.

The three kinds of instances were then presented in a categorization task. In all previous categorization tasks except one, which will be discussed below (Loftus, 1973), the category was always presented either before or simultaneously with the instance. When such a presentation strategy is used, both retrieval and comparison type models predict that low-typicality instances will be categorized more slowly than hightypicality instances, either because the low-typicality instance accesses a dissimilar description, or because it takes longer to access any description. However, if the instance is presented first, according to a retrieval model, it should provide the time for all instances to retrieve the information necessary for a categorization judgment.

Therefore, in order to distinguish atypical (i.e., low-typicality low-imagery) instances, in this task the instance was presented first. It remained on the screen until the subjects responded that they recognized its meaning. The first prediction made was that highimagery instances (e.g., robin, penguin) would be recognized more quickly than low-imagery instances (e.g., grackle) because the high-imagery instances are closely associated to descriptions and the low-imagery instances are not.

About $1 \mathrm{sec}$ after a subject responded that the instance was recognized, the category appeared and a category judgment was made. For this task, the following predictions were made. First, high-typicality instances (e.g., robin) should be categorized quickly because they are closely associated to the category and access descriptions similar to the category description. Second, unfamiliar instances (e.g., grackle) should be categorized quickly because they are only associated to the category, and hence, the category description should have been retrieved when the instance was recognized. Finally, atypical instances (e.g., penguin) should be categorized slowly because they are closely associated to a description that is not similar to the category description. Therefore, typical and unfamiliar instances (e.g., robin, grackle) should be categorized more quickly than atypical instances (e.g., penguin).

This prediction should hold only for the category that the instances are rated against (e.g., bird). There is no reason to assume that the instances would access other information in the same order. Therefore, as a control, each instance in the experiment was also categorized with respect to a more remote category of which it was also a member (e.g., animal, creature, organism). It was predicted that the categorization times of the different kinds of instances would not vary for the remote categories.

\section{Method}

Materials. A set of 84 items, with equal numbers of true and false sentences, was constructed for the experiment. The instances to be categorized were selected on the basis of typicality and imagery.

First, the typicality of approximately 20 instances from each of the categories was rated, using the following procedure. A group of 20 Rutgers undergraduates received a sheet with the following instructions: "On this page you will find pairs of words. The first word will be an instance of a category and the second word will be that category (e.g., apple-fruit). Please indicate how typical a member of the category the instance is by placing a number from 1 to 7 next to the pair. Place a 7 if it is the most typical instance you can imagine, and 1 if it is not a good instance at all. Please use as much of the scale as possibie and feel free to use any number as many times as you wish."

Next, a second group of 20 undergraduates rated the imagery of the instances on a scale from 1 to 7 , where 7 was most imageable and 1 was least imageable.

On the basis of these ratings, 21 instances were selected: 7 high-typicality high-imagery instances, 7 low-typicality high- 
Table 1

Mean Typicality and Imagery Ratings of the Types of Instances Used in Experiment 1

\begin{tabular}{llcl}
\hline & \multicolumn{3}{c}{ Type of Instance } \\
\cline { 2 - 4 } & Typical & Atypical & Unfamiliar \\
\hline Example & Goldfish & Eel & Herring \\
Typicality & 6.46 & 3.52 & 3.47 \\
Imagery & 6.74 & 5.95 & 2.79 \\
\hline
\end{tabular}

Note-The highest rating possible was 7 ; the lowest was 1 .

Table 2

True Instance-Category Pairs Presented in Experiment 1

\begin{tabular}{llllll}
\hline & \multicolumn{2}{c}{ Instance } & & \multicolumn{2}{c}{ Category } \\
\cline { 1 - 2 } Typical & Atypical & Unfamiliar & & Close & Remote \\
\hline Train & Blimp & Hovercraft & & Vehicle & Machine \\
Car & Sled & Jitney & & Vehicle & Machine \\
Tree & Mushroom & Philodendron & Plant & Organism \\
Collie & Dachshund & Foxhound & & Dog & Mammal \\
Goldfish & Eel & Herring & & Fish & Animal \\
Robin & Penguin & Grackle & Bird & Creature \\
Flower & Moss & Yucca & Plant & Organism \\
\hline
\end{tabular}

imagery instances, and 7 low-typicality low-imagery instances. The typicality and imagery ratings of these three groups are shown in Table 1 . The true items were constructed by pairing each instance with a "close" and a "remote" category. The close category was the category the instance had been rated against; the remote category was a larger category that contained the close one. All 42 true items are shown in Table 2. The false items were constructed by re-pairing the instances and categories used for the true items.

Procedure. The same apparatus was used as in previous experiments. Immediately after a brief warning flash, the instance appeared. It remained on the screen until the subject responded, or until $5 \mathrm{sec}$ elapsed. As soon as the subject recognized the meaning of the word, he pressed the right response button. If the subject did not know the meaning of the word, the left button was pressed. Approximately $1.2 \mathrm{sec}$ after the subject responded, a warning flash occurred, just prior to the appearance of the category. If the instance was a member of the category, the right button was pressed; otherwise, the left button was pressed. A new group of 22 subjects participated.

Subjects. In this experiment, as in the experiments that follow, the subjects were Rutgers undergraduates who participated to satisfy a course requirement. No subject ever participated in two experiments, or in two tasks within an experiment.

\section{Results and Discussion}

Subjects failed to respond less than $1 \%$ of the time and failures to respond were counted as errors. In this experiment, a hypothesis was tested about a few specific items that were carefully selected from a large initial pool of instances on the basis of their extreme scores along the dimensions of typicality and imagery. In order to demonstrate that all low-typicality instances were not alike, it was only necessary to demonstrate that the groups of instances within this sample differed. However, it was not clear how these results would generalize to any other instances that did not have extreme scores along the same dimension. Furthermore, the use of a small number of instances heavily weighted the odds against achieving a result that was significant at conventional levels of significance if both subjects and items were treated as random effects, even if the result was significant for the sample. Therefore, the use of quasi-F statistics was deemed inappropriate according to the criteria that Clark (1973) laid down for their use, and regular Fs were computed for each of the following experiments. However, for those who disagree with this argument or who are interested in generalizability, quasi$F$ ratios will be presented where they are substantially different from the regular Fs reported.

A total of $1 \%$ of the typical and atypical instances and $19 \%$ of the unfamiliar instances were not recognized. The instances that were recognized differed significantly from each other in recognition RT $[F(2,42)=54.32, p<.01]$. The mean recognition RT was $748 \mathrm{msec}$ for typical instances, $788 \mathrm{msec}$ for atypical instances, and $957 \mathrm{msec}$ for unfamiliar instances. Orthogonal contrasts revealed that unfamiliar instances were recognized more slowly than typical and atypical instances $[F(1,42)=54.63, p<.01]$, but atypical and typical instances did not differ significantly.

The rated imagery of the instances was correlated with their frequency $(r=.33)$ as measured by Kučera and Francis (1967). So rated imagery, typicality, frequency, and the log of the frequency were all used to predict recognition RT in a stepwise multiple-regression analysis to determine its best predictor. Imagery and recognition $\mathrm{RT}$ had the highest correlation $[\mathrm{r}=-.88$, $F(1,19)=64.1, p<.01]$. When imagery was entered into the regression equation, none of the correlations between the residual and any other variable approached significance. However, when the log of word frequency was forced in first, the correlation between the residual and word imagery was still significant $[\mathrm{r}=-.84$, $F(1,19)=4.16, p<.01]$. So rated imagery is the best predictor of recognition RT.

The mean true categorization RT and error rates for the three kinds of instances are shown in Table 3. Instances were categorized significantly more slowly $[F(1,21)=20.0, p<.01]$ as members of remote categories (e.g., creature, machine) than as members of close categories (e.g., bird, vehicle). Furthermore, as predicted, category closeness and instance type interacted significantly $[F(2,42)=6.13, p<.01]$. The

Table 3

Mean Reaction Time (RT) and Percent Error (E) for True Category Decisions in Experiment 1

\begin{tabular}{|c|c|c|c|c|c|c|}
\hline \multirow{3}{*}{$\begin{array}{c}\text { Category } \\
\text { Type }\end{array}$} & \multicolumn{6}{|c|}{ Instance Type } \\
\hline & \multicolumn{2}{|c|}{ Typical } & \multicolumn{2}{|c|}{ Atypical } & \multicolumn{2}{|c|}{ Unfamiliar } \\
\hline & RT & $\mathbf{E}$ & $R T$ & $\mathbf{E}$ & RT & E \\
\hline Close & 977 & 8 & 1077 & 9 & 1009 & 9 \\
\hline Remote & 1272 & 19 & 1137 & 22 & 1295 & 14 \\
\hline
\end{tabular}


categorization time for each item was also correlated with its frequency as measured by Kucera and Francis (1967). As anticipated, the correlation was not significant $(\mathrm{r}=.16)$, so those results cannot be accounted for by word frequency.

The general pattern of results of the categorization task also supports a mixed model in which typicality influences both retrieval and comparison. As predicted, the atypical instances (e.g., penguin) took longer to categorize for close categories than the typical (e.g., robin) and unfamiliar (e.g., grackle) instances. Also, as expected, this pattern did not hold for remote categories. Therefore, as predicted, there was a significant interaction between category closeness and instance typicality $[F(1,40)=24.58, p<.01]$. However, the unfamiliar instances were unexpectedly categorized somewhat more slowly than the typical instances, for close categories, although this result was not significant. Also unexpectedly, the atypical instances appeared to be categorized more quickly for the remote categories than the typical and unfamiliar instances. Orthogonal contrasts demonstrated that this result contributed to the critical interaction, since the component for close categories was not significant by itself. Hence, the results are not quite the strong and unambiguous support for the hypothesis that one would like. Also, a quasi- $F$ was performed on just the three close categories and it was not significant $\left[F^{\prime}(2,36)=3.02\right]$. Therefore, it was decided to replicate the results.

\section{EXPERIMENT 2}

According to the mixed model, in Experiment 1, the information that was retrieved about an instance before the category was presented facilitated the categorization of unfamiliar instances (e.g., grackle) and inhibited the categorization of atypical instances (e.g., penguin). However, subjects were given no special instructions about how to retrieve information. They were only told to respond when they recognized the instance. A variety of interpretations can be given to these instructions, which would increase subject variability and reduce the reliability of the predicted effect.

In Experiment 2, the same items and presentation procedure were used, but more specific instructions were given. When the instance appeared, subjects were told to make an image of it and to respond as soon as the image was made. They were to hold the image until the category appeared and then make a category judgment. It was assumed that generating an image would require the same information that was relevant to a category judgment. Therefore, the predictions were the same as in Experiment 1.

First, it was predicted that images would be generated faster for the high-imagery instances (e.g., robin, penguin) than for the low-imagery instances (c.g., grackle). Second, since a typical instance such as "robin" presumably looks like a bird, generating an image of a robin should require the retrieval of information useful for categorizing it as a bird. Similarly, if the only thing known about an unfamiliar instance such as "grackle" is that it is a bird, then a subject should have to access that fact in the attempt to make an image of a grackle. Furthermore, what should the image of a grackle look like? It presumably closely resembles the image of a typical bird, since there is no information directly associated with "grackle" to modify the general properties accessed from "bird." Therefore, a grackle should also be categorized quickly. Finally, since a penguin does not look like a bird, the information accessed in generating an image of it should inhibit rather than facilitate its categorization as a bird. Therefore, for close categories, typical and unfamiliar instances should again be categorized faster than atypical instances. However, for remote categories, there is no reason to think that a robin or a grackle looks more like a creature than a penguin does. Therefore, for remote categories, the prediction was again that there should be no differences between the three instance types.

\section{Method}

The material, apparatus, and procedure were identical to Experiment 1 . The only difference was a change in part of the subjects' instructions. Subjects were told that when the instance appeared they were to make an image of it, and that as soon as they made the image they were to respond by pushing the right response button. They were to maintain the image until the category appeared. Approximately $1.2 \mathrm{sec}$ later, the category appeared, and subjects made the appropriate category response. A total of 21 subjects participated.

\section{Results and Discussion}

Less than $1 \%$ of the time, subjects failed to respond. These failures to respond were counted as errors. Images were generated most quickly for typical instances such as "robin" ( $999 \mathrm{msec})$, second most quickly for atypical instances such as "penguin" (1,045 $\mathrm{msec})$, and least quickly for unfamiliar instances such as "grackle" $(1,190 \mathrm{msec}) \quad[\mathrm{F}(2,40)=8.2, \quad \mathrm{p}<.01]$. Orthogonal contrasts revealed that unfamiliar instances were recognized more slowly than typical and atypical instances $[F(1,42)=8.74, p<.01]$, but atypical and typical instances did not differ significantly. Hence, as expected, the image-generation RT mirrored the recognition RT recorded in Experiment 1.

Again, imagery, typicality, frequency, and the log of frequency were entered into a stepwise multipleregression analy sis to predict recognition RT. Again, when imagery entered the equation $[\mathrm{r}=-.64, \mathrm{~F}(1,19)=13.5$, $p<.01]$, none of the correlations between the other variables and the residual approached significance. Again, when the $\log$ of frequency was forced in first, the correlation between the residual and imagery remained significant $[r=-.52, F(1,19)=6.69, p<.025]$. So 
Table 4

Mean Reaction Time (RT) and Percent Error (E) for True Category Decisions in Experiment 2

\begin{tabular}{|c|c|c|c|c|c|c|}
\hline \multirow{3}{*}{$\begin{array}{c}\text { Category } \\
\text { Type }\end{array}$} & \multicolumn{6}{|c|}{ Instance Type } \\
\hline & \multicolumn{2}{|c|}{ Typical } & \multicolumn{2}{|c|}{ Atypical } & \multicolumn{2}{|c|}{ Unfamiliar } \\
\hline & $\mathrm{RT}$ & $\mathbf{E}$ & $\mathrm{RT}$ & $\mathrm{E}$ & $\mathrm{RT}$ & $\mathrm{E}$ \\
\hline $\begin{array}{l}\text { Close } \\
\text { Remote }\end{array}$ & $\begin{array}{l}1156 \\
1242\end{array}$ & $\begin{array}{r}8 \\
12\end{array}$ & $\begin{array}{l}1241 \\
1469\end{array}$ & $\begin{array}{l}10 \\
22\end{array}$ & $\begin{array}{l}1177 \\
1273\end{array}$ & $\begin{array}{l}17 \\
24\end{array}$ \\
\hline
\end{tabular}

again, imagery was the best predictor of recognition RT.

The mean true categorization RTs and error rates for the three types of instances are shown in Table 4. As predicted for close categories, the pattern of results was similar to Experiment 1. As can be seen in Table 4, again the atypical instances (e.g., penguin) were categorized more slowly than typical (e.g., robin) and unfamiliar (e.g., grackle) instances for close categories (e.g., bird). However, unlike Experiment 1, the same RT pattern occurred for remote categories (e.g., creature), so that there was a significant main effect of instance type $[F(2,40)=9.93, p<.01]$. However, when a quasi-F was again computed for the effect of instance type for just the close categories, it was again nonsignificant $[F(2,28)=2.92]$. There was also a significant main effect of category closeness $[F(1,20)=16.81$, $\mathrm{p}<.01]$.

Considered together, the results of Experiments 1 and 2 provide a consistent picture of instance categorization in this task. In both experiments, the same pattem of RT was obtained for the close category condition. As predicted, atypical instances were categorized more slowly than typical and unfamiliar instances. No difference between the three instance types was predicted for the remote categories. In the two experiments, only the atypical instances departed from this prediction. But they departed in opposite directions in the two experiments. Therefore, it seems reasonable to conclude that there were no reliable differences in RT found between instance types for remote categories, and the only reliable difference observed was the predicted one.

These results are inconsistent with either a simple retrieval model or a simple comparison model. Each simple model can account for the results of only one kind of low-typicality instance. A retrieval model can naturally account for the findings with unfamiliar instances (e.g., grackle). As it would predict, it takes longer to recognize and generate images of unfamiliar instances, but if sufficient time is allowed for the retrieval of this information, the instances are categorized as quickly as typical instances. However, even if sufficient time is given for retrieval for atypical instances (e.g., penguin), these instances still take longer to verify than the other two kinds of instances.

Conversely, a simple comparison model can account for the categorization of atypical instances but not for unfamiliar instances. The comparison model explains the slow categorization of atypical instances by the assumption that the description of an atypical instance is dissimilar to a description associated with the category. There is supportive evidence for this assumption (e.g., Rosch, Mervis, Gray, Johnson, \& Boyes-Baem, 1976), and it seems a plausible one for some instances. However, a model in which all RT differences are due to the similarity of descriptions simply does not predict the existence of unfamiliar instances such as "grackle." In order to account for the fact that they take longer to recognize, but once recognized take no longer to categorize, than typical instances, additional assumptions have to be added about retrieval. The combination of retrieval and comparison assumptions results in a mixed model, in which a categorization decision is influenced by both the kind of information accessed by the instance and the speed with which it is accessed.

Finally, let us consider what information in a semantic description is used for making a category judgment. A property-list comparison model (McCloskey \& Glucksberg, in press; Schaeffer \& Wallace, 1970; Smith et al., 1974) would explain the results of Experiments 1 and 2 by assuming that typical instances access property lists similar to the category property list, atypical instances access property lists dissimilar to the category property list, and unfamiliar instances only access the category property list itself. Therefore, during the comparison stage, the category property list would be compared to a similar property list for typical instances, and these instances would therefore be categorized rapidly; the category property list would be compared to itself for unfamiliar instances, so they would be categorized rapidly; but the category property list would be compared to a dissimilar list for atypical instances, so these would be categorized more slowly. A category label model would explain the same results by assuming that typical and unfamiliar instances are most closely associated to the same category label (e.g., "bird" for "robin" and "grackle"), but that atypical instances are most closely associated to another category label (e.g., "animal" for "penguin"). Therefore, during the delay between the presentation of the instance and the category, the appropriate category label would be rapidly retrieved from typical instances and less rapidly retrieved from unfamiliar instances. However, an inappropriate category label would be retrieved from atypical instances.

A choice between the role of property lists and category labels cannot be made on the basis of the first two experiments in this study, since both of the models just described are consistent with their results. However, there is another study whose results support category label retrieval. Loftus (1973) found that the frequency of production of the category as a response to the instance was correlated with the time to categorize the instance, when the instance was presented first. The more frequently the category was produced, the 
quicker the categorization. On the other hand, this correlation does not prove that production of the appropriate category was the causal factor making unfamiliar instances faster than atypical ones. Furthermore, the fact that the same results were obtained under imagery instructions as under reccgnition instructions might tend to favor a property-list model, since the construction and maintenance of an image requires the retrieval of perceptual properties but does not necessarily require the retrieval of a category label. However, in Experiment 2, nothing prevented subjects from retrieving category labels in addition to generating images. Therefore, Experiment 3 was done to examine the effect of imagery uncontaminated by extraneous associations.

\section{EXPERIMENT 3}

The similarity of the results of Experiments 1 and 2 could be obtained for one of two reasons. Either subjects did the equivalent of generating images in Experiment 1, even though they were not instructed to do so, or they generated category labels in Experiment 2, even though they were not instructed to do so. Experiment 3 was a replication of Experiment 2 in which greater care was taken that subjects would generate images and do nothing more. Besides generating an image, subjects were required to count down from a random three-digit number until the category was presented. This procedure was adopted in order to reduce the probability that they were consciously generating category labels during the interval between the presentation of the instance and the presentation of the category.

\section{Method}

The experiment was in all respects identical to Experiment 2, except for the following. When the instance appeared, to the right of it was a three-digit number, randomly selected from the range 200-999. The subject was instructed to generate the image and, as soon as the image was generated, to press the right response button and to simultaneously begin counting down, out loud, from the number. If the subject did not know what the instance meant, the left response button was to be pushed. The subject was told to maintain the count and image until the category was presented, at which point, the categorization task was to be performed as rapidly as possible. A total of 19 subjects participated.

\section{Results and Discussion}

Subjects failed to respond less than $1 \%$ of the time, and failures to respond were counted as errors. The subjects failed to recognize or make an image of less than $1 \%$ of the typical instances, $3 \%$ of the atypical instances, and $26 \%$ of the unfamiliar instances. For the remaining instances, images were generated for the typical instances in $1,317 \mathrm{msec}$, for the atypical instances in $1,366 \mathrm{msec}$, and for unfamiliar instances in $1,627 \mathrm{msec}$. These generation times differ significantly $\{\mathrm{F}(2,36)=34.22, \quad \mathrm{p}<.01]$. Orthogonal contrasts
Table 5

Mean Reaction Time (RT) and Percent Error (E) for True Category Decisions in Experiment 3

\begin{tabular}{|c|c|c|c|c|c|c|}
\hline \multirow{3}{*}{$\begin{array}{c}\text { Category } \\
\text { Type }\end{array}$} & \multicolumn{6}{|c|}{ Instance Type } \\
\hline & \multicolumn{2}{|c|}{ Typical } & \multicolumn{2}{|c|}{ Atypical } & \multicolumn{2}{|c|}{ Unfamiliar } \\
\hline & RT & $\mathrm{E}$ & RT & $\mathrm{E}$ & RT & $\mathbf{E}$ \\
\hline Close & 1293 & 8 & 1489 & 11 & 1313 & 13 \\
\hline Remote & 1465 & 20 & 1540 & 32 & 1535 & 19 \\
\hline
\end{tabular}

revealed that images were generated more slowly for unfamiliar than for typical and atypical instances $[F(1,36)=39.51, p<.01]$, but the image-generation times for typical and atypical instances did not differ significantly.

Again, imagery, typicality, frequency, and the log of frequency were entered into a stepwise multipleregression analysis to predict recognition RT. Again, when imagery entered the equation $[\mathrm{r}=-.82, \mathrm{~F}(1,19)=39.0$, $p<.01$ ], none of the correlations between the other variables and the residual approached significance. Again, when the log of the frequency was forced in first, the correlation between the residual and imagery remained significant $[r=-.77, F(1,19)=25.9, p<.01]$. So again, imagery was the best predictor of recognition RT.

The true mean categorization RTs and error rates for the three types of instances are shown in Table 5 . As can be seen from the table, the pattem of results was similar to Experiments 1 and 2. Orthogonal contrast revealed that for close categories, atypical instances (e.g., penguin) were categorized more slowly than unfamiliar instances (e.g., grackle) $[F(1,36)=10.19, p<.01]$, but unfamiliar and typical instances did not differ in their categorization times. However, again when a quasi- $F$ was used to assess the effect of instance typicality for the close categories, it was nonsignificant $\left[F^{\prime}(2,43)=2.93\right]$. There were no significant differences between instance types for remote categories. Also, again, decisions were made faster for close than for remote categories $[F(1,18)=7.12, p<.025]$. These results strongly suggest that the effect of imagery instructions is equivalent to simply giving subjects sufficient time to recognize instances before they categorize them. This could mean either that people spontaneously generate images when not instructed to do so, or that generating an image of an instance has no influence on categorizing it.

The task was quite demanding, and it is therefore unlikely that there was much conscious processing besides the image generation and counting. However, it is possible that the instances automatically "primed" categories without conscious effort on the part of the subject. The existence of an automatic priming effect is well established (Neely, 1976). However, whether a purely automatic priming effect could have the magnitude of the difference observed between atypical 
and unfamiliar instances in this experiment is not known. At a minimum, an automatic priming interpretation cannot be ruled out.

\section{GENERAL DISCUSSION}

The results of the experiments in this study strongly suggest that differences in both the retrieval and the comparison of semantic descriptions contribute to the observed RT differences between the high- and lowtypicality instances in a categorization task. While the results of these experiments cannot be generalized beyond the specific items used here to predict how other instances will be categorized, the fact that atypical and unfamiliar instances differed significantly in recognition and categorization $R T$ is sufficient to demonstrate that all low-typicality instances do not form a homogeneous group.

While these results shed light on the contributions of retrieval and comparison to RT differences, they are equally compatible with both category label and property-list models of categorization that incorporate the appropriate assumptions about what information is associated to the different instance types. Is there, then, any way of deciding which kind of information a category judgment is based on?

One test of a model is how well it fits with models in related areas. When what is known about categorization and memory in general is considered, a categorization model that incorporates both properties and category labels appears plausible (Collins \& Loftus, 1975). For, at an intuitive level, property-list comparison and category label retrieval both model fundamental ways in which we know what something is. If we go to the acquarium and see two new sea creatures, we recognize a mackeral as a fish because it looks like a fish (property comparison); we recognize a whale as a mammal because we have been told that it is a mammal (category labeling). Furthermore, the existence of the representations that necessarily underlie these two ways of categorizing has been demonstrated, al though the connection to categorization is usually unstated. Prototype-generation studies provide evidence for property lists, while paired associate and list-learning studies show how instances and categories may become associated.

In conclusion, there seems little reason to doubt the richness of the semantic representation or the variety of strategies for extracting information from it. What is needed now is the development of new experimental techniques for the explication of this structure in detail.

\section{REFERENCES}

Clark, H. H. The language-as-fixed-effect fallacy: A critique of language statistics in psychological research. Journal of Verbal Learning and Verbal Behavior, 1973, 12, 335-359.

Collins, A. M., \& Loftus, E. F. A spreading activation theory of semantic processing. Psychological Review, 1975. 82, 407-428.

Collins, A. M., \& Quillian, M. R. Retrieval time from semantic memory. Joumal of Verbal Learning and Verbal Behavior, 1969, 8, 240-248.

Glass, A. L., \& Holyoak, K. J. Alternative conceptions of semantic memory. Cognition, 1975, 3, 313-339.

KuČERA, M., \& FrANCIs, W. N. Computational analysis of present-day American English. Providence, R.I: Brown University Press, 1967.

LofTus, E. F. Category dominance, instance dominance, and categorization time. Journal of Experimental Psychology, 1973, 97, 70-94.

McCloskey, M., \& Glucksberg, S. Decision processes in verifying class inclusion statements: Implications for models of memory. Cognitive Psychology, in press.

NEELY, J. H. Semantic priming and retrieval from lexical memory: Evidence for facilitatory and inhibitory processes. Memory \& Cognition, 1976, 4, 648-654.

Rips, L. J., Shopen, E. J., \& Smith, E. E. Semantic distance and the verification of semantic relations. Journal of Verbal Leaming and Verbal Behavior, 1973, 12, 1-20.

Rosch, E. R. On the internal structure of perceptual and semantic categories. In T. M. Moore (Ed.), Cognitive development and the acquisition of language. New York: Academic Press, 1973.

Rosch, E., Mervis, G. B., Gray, W., Johinson, D., \& Boyes-BaEm, P. Basic objects in natural categories. Cognitive Psychology, 1976, 8, 382.440.

Schaeffer, B., \& Wallace, R. The comparison of word meanings. Journal of Experimental Psychology, 1970, 86, 144-152.

Smrth, E. E., Shopen, E. J., \& Rips, L. J. Structure and process in semantic memory: A featural model for semantic decisions. Psychological Review, 1974, 81, 214-241.

(Revision accepted for publication September 11, 1978.) 